\title{
A!
}

This is an electronic reprint of the original article.

This reprint may differ from the original in pagination and typographic detail.

Huan, Siqi; Ajdary, Rubina; Bai, Long; Klar, Ville; Rojas, Orlando J.

\section{Low Solids Emulsion Gels Based on Nanocellulose for 3D-Printing}

\section{Published in:}

Biomacromolecules

DOI:

10.1021/acs.biomac.8b01224

Published: 11/02/2019

Document Version

Peer reviewed version

Published under the following license:

Unspecified

Please cite the original version:

Huan, S., Ajdary, R., Bai, L., Klar, V., \& Rojas, O. J. (2019). Low Solids Emulsion Gels Based on Nanocellulose for 3D-Printing. Biomacromolecules, 20(2), 635-644. https://doi.org/10.1021/acs.biomac.8b01224

This material is protected by copyright and other intellectual property rights, and duplication or sale of all or part of any of the repository collections is not permitted, except that material may be duplicated by you for your research use or educational purposes in electronic or print form. You must obtain permission for any other use. Electronic or print copies may not be offered, whether for sale or otherwise to anyone who is not an authorised user. 


\title{
Low solids emulsion gels based on nanocellulose for 3D-printing
}

\author{
Siqi Huan ${ }^{1}$, Rubina Ajdary ${ }^{1}$, Long Bai ${ }^{1, *}$, Ville Klar ${ }^{2}$, Orlando J. Rojas ${ }^{1, *}$ \\ ${ }^{1}$ Department of Bioproducts and Biosystems, School of Chemical Engineering, Aalto University, \\ P.O. Box 16300, FIN-00076 Aalto, Espoo, Finland. \\ ${ }^{2}$ Department of Mechanical Engineering, School of Engineering, Aalto University, P.O. Box \\ 16300, FIN-00076 Aalto, Espoo, Finland.
}

\begin{abstract}
Multiphase (emulsion) gels with internal phase fractions between 0.1 and 0.5 were formulated at low loadings of cellulose nanofibrils (CNF), alginate and polylactide (PLA). Their properties (rheology and morphology) fitted those of inks used for direct ink writing (DIW). The effect of formulation and composition variables were elucidated after printing cubic scaffolds and other solid designs. The distinctive microstructures that were developed allowed high printing fidelity and displayed limited shrinkage after room temperature and freeze drying $(0$ and $5 \%$ shrinkage in the out-of-plane and in-plane directions upon freeze drying, respectively). The CNF added in the continuous phase was shown to be critical to achieve rheology control, as an effective interfacial stabilizer and to ensure the printability of the ink toward high structural reliability.

We found that the extent of shape retention of the dried scaffolds resulted from the tightly locked internal structure. The PLA that was initially added in the non-polar or organic phase (0 to
\end{abstract}


$12 \%$ ), was randomly embedded in the entire scaffold, providing a strong resistance to shrinkage during the slow water evaporation at ambient temperature. No surface collapse or lateral deformation of the dried scaffolds occurred, indicating that the incorporation of PLA limited drying-induced shape failure. It also reduced compression strain by providing better CNF skeletal support, improving the mechanical strength.

Upon re-wetting, the combination of the hydrophilicity imparted by CNF and alginate together with the highly porous structure of the 3D material and the internal micro-channels contributed to high water absorption via capillary and other phenomena (swelling \% between 400 and $900 \%$ ). However, no shape changes occurred compared to the initial 3D-printed shape. The swelling of the scaffolds correlated inversely with the PLA content in the precursor emulsion gel, providing a means to regulate the interaction with water given its low surface energy.

Overall, the results demonstrate that by compatibilization of the CNF-based hydrophilic and the PLA-based hydrophobic components, it is possible to achieve shape control and retention upon 3D printing, opening the possibility of adopting low-solids inks for DIW into dry objects. The dryable CNF-based 3D structural materials absorb water while being able to support load (high elastic modulus) and maintain the shape upon hydration.

Keywords: 3D printing, emulsion gel, cellulose nanofibrils, alginate, poly(lactic acid), direct ink writing (DIW).

\section{INTRODUCTION}

3D printing is a popular and customizable method to fabricate structures for application in the materials, chemical, and biology fields. ${ }^{1-4}$ The mainstream commercial systems currently available are designed to process metal, ceramics and thermoplastics. ${ }^{5}$ In contrast, biobased materials have 
been adopted only to a limited extent, especially when designing structural materials. ${ }^{6}$ Fluids that display viscous flow under shear stress (shear thinning) and rapid recovery after relaxation (shapefixing) are conveniently used as 3D printable inks. ${ }^{7}$ They transform from gel-like, over liquid-like and back to gel-like upon extrusion. ${ }^{8}$ Direct ink writing (DIW) is an extrusion-based 3D printing technique that enables continuous layer-by-layer asemblies ${ }^{9}$ with a variety of inks that includes colloidal gels ${ }^{10}$ concentrated polyelectrolyte complexes, ${ }^{11}$ and hydrogels. ${ }^{12}$ Recently, emulsions and foams have been introduced for such purposes. ${ }^{13,14}$ However, the formulations of emulsions and foams require high solid concentration to ensure stable printing toward high-fidelity shapes. ${ }^{15}$ This fact has limited the adoption of biobased components for DIW, which otherwise are interesting alternatives to inorganic and synthetic counterparts. ${ }^{16}$ Owing to their tendency to form hydrogels, cellulose nanofibrils (CNF) are ideally suited for related purposes, ${ }^{17,18}$ as has been discussed in recent reports. ${ }^{19-21}$ Still, the weak gelation strength at low concentration of noncrosslinked CNF prevents a broader use..$^{22,23}$

The incorporation to CNF of auxiliary hydro-soluble polymers, such as alginate, has been shown as an efficient way to solidify the system. ${ }^{24-26}$ However, the loss of structure upon drying remains a major challenge. ${ }^{27}$ Indeed, the initial shape of printed CNF hydrogels is easily lost at ambient temperature drying and even collapse or shrink upon freeze-drying, especially when used at low concentrations. ${ }^{20}$ The incorporation of other polymers may provide a route to enhance the performance of CNF-based printed objects if they can support or retain the shape. Obviously, such possibility is not practical if printability is impaired or prevented ${ }^{28}$ or if the interfacial compatibility with CNF is lacking, a typical situation in the case of non-polar polymers. ${ }^{29}$ The incorporation of the latter, however, can be beneficial in efforts to limit the sensitivity to water. For this purpose, a viable alternative is the use of multiphase, emulsion systems. ${ }^{30}$ 
Oil-in-water emulsions can incorporate $\mathrm{CNF}$ at the interface ${ }^{31}$ or in the continuous phase ${ }^{32}$ the addition of non-polar polymers in such emulsions has not been widely explored,$^{33}$ especially if high polymer loadings are desired, such as in high-internal-phase emulsions. Herein, we propose a single-step synthesis of high fidelity DIW inks based on emulsion gels based on CNF in the presence of alginate and poly(lactic acid) (PLA). The non-adsorbing CNF is dispersed in the aqueous phase as a gel-like network and to act as co-stabilizer of emulsified PLA droplets. ${ }^{34}$ Alginate is introduced in the continuous phase as auxiliary component to improve the weak strength of the emulsified gel, via $\mathrm{Ca}^{2+}$-mediated post-solidification of the printed objects. We demonstrate no surface collapse nor shape deformation upon room-temperature drying, offering a new pathway for the design and fabrication of biobased, moldable 3D printing inks. Moreover, we show the possibility of no or limited structural changes upon re-hydration of the dried 3D-printed designs. By compatibilization of the hydrophilic and hydrophobic components via emulsification, we achieve control on swelling and open the possibility for adopting low-solids CNF-based emulsion inks. Such systems can be printed into tailorable structures that have been rarely reported. More importantly, to the best of our knowledge, this is the first report to utilize CNF-based emulsion gels with hydrophobic PLA to reveal precise printing toward dryable and rehydratable 3D objects.

\section{EXPERIMENTAL SECTION}

Materials. Polylactide (PLA, 6060D, $\left.M_{w}=191000\right)$ was obtained from Nature Works, United States. Span 80, Tween 80, sodium alginate (A2033, medium viscosity, Sigma-Aldrich), calcium chloride $\left(\mathrm{CaCl}_{2}\right)$, Nile red and chloroform $\left(\mathrm{CHCl}_{3}\right)$ were purchased from Sigma-Aldrich (Helsinki, Finland) and used as received. Milli-Q water was purified with a Millipore Synergy UV unit and used throughout the experiments. Cellulose nanofibrils (CNF) were prepared by disintegration of 
never-dried, fully bleached and fines-free sulfite hardwood (birch) fibers obtained from a Finnish pulp mill, as described previously. ${ }^{35}$ The fibers were disintegrated through a high-pressure microfluidizer (M110P, Microfluidics Int. Co., Newton, MA) using six passes to obtain a CNF suspension in water at $1.8 \mathrm{wt} \%$ solids. The average width of the cellulose nanofibrils was $\sim 20 \pm$ $8 \mathrm{~nm},{ }^{34}$ and the surface anionic charge content was $\sim 0.04 \mathrm{mmol} / \mathrm{g} .{ }^{31}$

Emulsion gel. The CNF suspension was mixed with alginate solution for a total solids content of $3 \mathrm{wt} \%$ with respect to the mass of the aqueous phase (1.5\% based on the emulsion). The nomenclature used for the inks (emulsion gels) and the respective dry mass ratio of $\mathrm{CNF} /$ alginate and PLA is summarized in Table S1. Preliminary studies indicated that by adding the same amounts of CNF and alginate was optimal for emulsion gel printing, following casting and crosslinking tests, Figure S1 and S2. A surfactant mixture containing Tween 80 and Span 80 (3:1, $2 \mathrm{wt} \%$ ) was dissolved into $\mathrm{CNF} /$ alginate aqueous suspension before emulsification. The organic phase consisted of PLA dissolved in chloroform, $\mathrm{CHCl}_{3}$. Herein, the PLA weight $\%$ is reported relative to the mass of the organic phase (between 0 and $12 \%$ ). To prepare the emulsions, a given volume of the CNF suspension was mixed with alginate and surfactant solution followed by 5 -min sonication in a water bath. Solutions of given concentrations of PLA in the organic phase were blended with the aqueous, continuous phase using a high-speed mixer operated at $10000 \mathrm{rpm}$ for 2 min (Digital UltraTurrax, IKA, Germany). Two composition variables were considered in the preparation of the emulsion gels: (a) PLA concentration from 0 to $12 \mathrm{wt} \%$ at fixed volume fraction of the organic phase $f_{0}=0.2$ (or water-to-oil ratio WOR $=80: 20$ ) and, (b) $f_{\mathrm{o}}$ from 0.1 to 0.5 (WOR from 90:10 to 50:50) at fixed PLA concentration of $12 \mathrm{wt} \%$. After preparation, the emulsions were equilibrated and stored at ambient temperature for $24 \mathrm{~h}$ before characterization and 3D printing. 
Emulsion morphology and rheology. The emulsion droplets were observed with an optical microscope (Leica DM 750, Leica, Germany) with $20 \times$ objective lens. A drop of the emulsion gel was placed onto a microscope slide and covered with a glass coverslip (Assistent, Sondheim, Germany). The emulsion droplets were also examined with a confocal laser scanning microscope (CLSM) with a $63 \times$ oil immersion objective lens (Leica DMRXE, Leica, Germany). The oil or organic phase was dyed with $10 \mu \mathrm{L}$ of Nile red solution $(1 \mathrm{mg} / \mathrm{mL}$ ethanol) added to $100 \mu \mathrm{L}$ sample (the excitation and emission spectrum for Nile red are $488 \mathrm{~nm}$ and $539 \mathrm{~nm}$, respectively). After homogeneously mixing with a pipette, $6 \mu \mathrm{L}$ of dyed sample was placed on a microscope slide and covered with a glass coverslip. The coverslip was quickly fixed with a nail polish to avoid evaporation.

Rheology. The shear viscosity of the emulsion gels was measured with a rheometer (MCR 302, Anton Paar, Germany) using parallel plates (PP25) with a gap fixed at $0.5 \mathrm{~mm}$. All samples were pre-sheared at $10 \mathrm{~s}^{-1}$. The apparent shear viscosity was monitored by increasing the shear rate from 0.01 to $100 \mathrm{~s}^{-1}$. For dynamic viscoelastic measurements, the linear viscoelastic range was determined with a strain sweep $(0.01$ to $100 \%)$ at a fixed frequency of $10 \mathrm{rad} / \mathrm{s}$. After this, a dynamic frequency sweep was conducted using a parallel plate geometry with a gap fixed at 0.5 $\mathrm{mm}$ and by applying a constant strain of $0.5 \%$, which was within the linear region, and over a frequency range between 0.1 and $100 \mathrm{rad} / \mathrm{s}$. The dynamic mechanical spectra were obtained by recording the storage $\left(G^{\prime}\right)$ and loss $\left(G^{\prime \prime}\right)$ moduli as a function of frequency. All measurements were performed at $25^{\circ} \mathrm{C}$.

3D Printing of the emulsion gel. A 3D-printer prototype was built in-house with the purpose of extruding paste-like materials. The operation window is similar to that of commercial units such as nScrypt systems (3Dn-300, nScrypt Inc., USA). The system utilized a simple syringe pump 
system with disposable $10 \mathrm{ml}$ syringe (Norm-Ject, Air-Tite Products Co., Inc., USA) and tapered tips (Drifton, Denmark). The nozzle size of the tip used was $0.63 \mathrm{~mm}$. The moving speed of the print head was $7.5 \mathrm{~mm} / \mathrm{s}$, and the optimized extrusion speed was set at $0.012 \mathrm{~mm} / \mathrm{s}$ based on primary tests of the inks. Cubic grid scaffolds $(2 \mathrm{~cm} \times 0.5 \mathrm{~cm}$ length and width) were printed as well as specially designed shapes. The printed layers were built with parallel lines at $45^{\circ}$ from the edges of the cube ensuring that the alternating layers were oriented perpendicular to each other. After printing, the produced scaffolds were gently moved to crosslinking solution ( $1 \mathrm{wt} \%$ aqueous $\mathrm{CaCl}_{2}$ ) for $\sim 24 \mathrm{~h}$ before characterization. Note: once dried and upon immersion in water (rehydration), a slight increase of line diameter in the printed objects occurred due to the swelling of CNF.

Scaffolds structure and swelling. Microstructure. The microstructure of the lines in the printed scaffolds, after drying, was revealed by a scanning electron microscopy (SEM, Zeiss Sigma VP, German) operated under vacuum and at an accelerated voltage of $2 \mathrm{kV}$. The internal structure was revealed from clean knife cuts. The surface and inner sections of the scaffolds were sputtercoated with platinum before imaging.

Shrinking and swelling of $3 D$ printed scaffolds. The visual appearance and dimensions (plane and out-of-plane directions) of the printed cubic scaffolds after crosslinking and drying were recorded. A ruler was used as a reference in the images. For comparison, dimensional changes were measured after drying at room temperature (RT) and freeze-drying. In the former case, the scaffolds were imaged after crosslinking. For the freeze-dried materials, imaging was carried out with the cross-linked scaffolds that were vacuum dried $(>48 \mathrm{~h})$ after freezing overnight $\left(-18{ }^{\circ} \mathrm{C}\right)$. The volumetric shrinkage was calculated according to the size variation from the hydrated to the dried condition. 
The freeze-dried scaffolds were immersed in water (re-hydration) and the extent of swelling was determined. The water absorbed was recorded gravimetrically at given time intervals. Before weighing, excess water was removed gently by using filter paper. The experiments were carried out at room temperature (three replicates were used). The $\%$ swelling ratio $(\mathrm{g} / \mathrm{g})$ was calculated according to: ${ }^{36}$

Swelling ratio $=\frac{W_{2}-W_{1}}{W_{1}} \times 100$

where $W_{1}$ is the initial weight of dried sample, and $W_{2}$ is the weight after soaking in water at the given time.

\section{RESULTS AND DISCUSSION}

Structure of the emulsion gel. A conceptual schematic illustration of the procedure used to prepare the CNF-based emulsion gels (inks) is shown in Figure 1a. The continuous, gel-like phase contained non-interacting CNF and alginate, both of which are negatively charged and therefore are subjected to electrostatic repulsion. The rheological properties of the ink resulted from the highly entangled fibril network formed by CNF at the given concentration (1.5 wt\%) (Figure 1b). ${ }^{18}$ The surfactant mixture was used to stabilize the PLA solution (organic phase). As shown in the optical image (Figure 1c), PLA droplets were dispersed in the emulsion gel, a multiphase system proposed as efficient route to compatibilize the hydrophobic PLA biopolymer with the hydrophilic CNF. The limited flocculation that occurred in the system was induced by depletion effects of the non-adsorbing CNF and alginate (Figure 1d) ${ }^{37}$ The stability of the emulsion droplets is explained by depletion stabilization mechanisms. ${ }^{34}$ These results also demonstrate that the developed emulsion gel is a robust system that facilitates the integration of components with differing surface energies; related biobased, multiphase systems can be expanded or generalized 
for the formulation of other DIW inks, e.g., containing polymers, biodegradable or not, to endow printed objects with given functions, such as bio-absorbability, anti-microbial, conductive (thermal, electric) and magnetic, for example).

(a) Separate oil \& aqueous phases

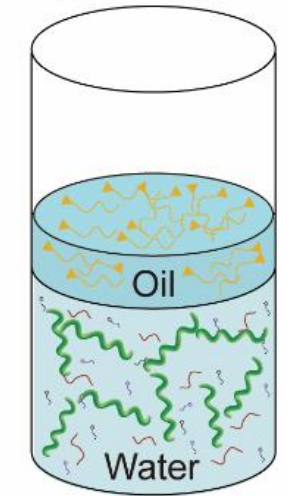

(b)

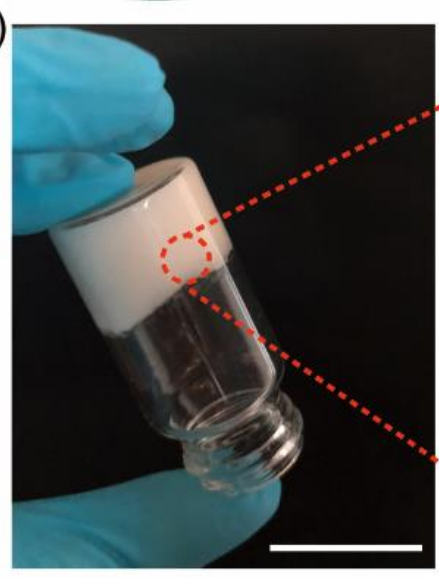

(c)

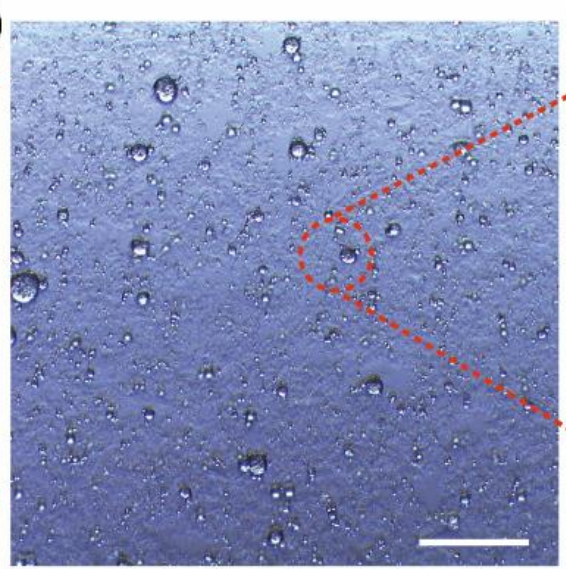

Emulsion gel
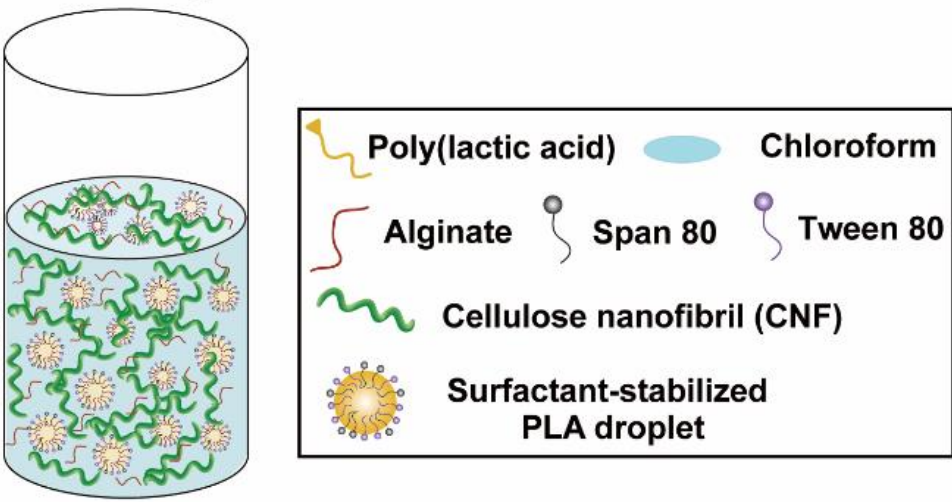

(d)

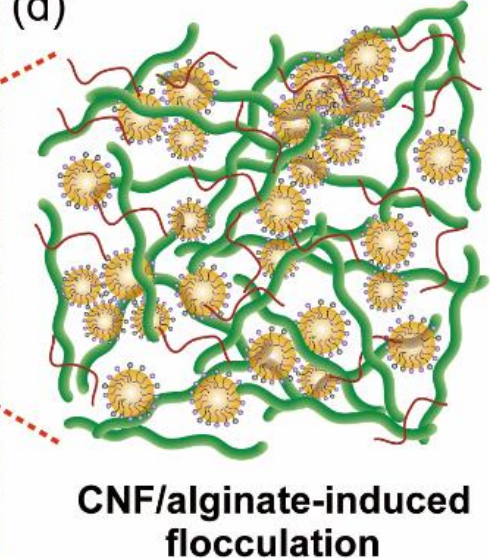

Figure 1. (a) Schematic illustration (not to scale) of the preparation of CNF/alginate-stabilized emulsion gels containing PLA and nonionic surfactants. The concentration of CNF and alginate is equal in all ink formulations. (b) Visual appearance of formed emulsion gel stored in an inverted vial. The emulsion was prepared with an oil fraction of 0.3 (water-to-oil ratio, WOR $=70: 30$ ) and PLA concentration of $12 \mathrm{wt} \%$. The emulsion gel was stored in ambient conditions for $24 \mathrm{~h}$ (scale bar $=2.5 \mathrm{~cm}$ ). (c) Optical images of the formed emulsion gel showing some larger droplets produced after depletion flocculation (scale bar $=100$ $\mu \mathrm{m})$. (d) Proposed component distribution of the emulsion gel containing PLA droplets.

The microstructured emulsion gels were prepared at $f_{o}=0.2$ and varying PLA concentrations in the organic phase, Figure 2a. The low viscosity of this phase facilitated emulsification into 
small droplets that were stabilized by the surfactant mixture. At PLA concentration of 0 and $4 \mathrm{wt} \%$, the emulsion droplets were homogeneously dispersed (diameter of $1 \sim 4 \mu \mathrm{m}$ ) in the aqueous phase (Figure 2 $\mathbf{a}_{1}$ and $\mathbf{a}_{2}$ ). The presence of CNF enabled a gel-like continuous phase that prevented PLA droplet creaming and coalescence (Figure $2 \mathbf{a}_{1}$ and $\mathbf{a}_{2}$ ). With increasing PLA loading to $8 \mathrm{wt} \%$, the droplet diameter significantly increased and became polydispersed (Figure 2a 3 ), which was likely due to reduced emulsifying efficiency. The low efficiency of sonication was mainly caused by the high viscosity of dispersed phase in this condition. Despite the restricted droplet transport in the emulsion gel, droplets coalescence occurred at PLA concentration of $12 \mathrm{wt} \%$ (see the large and nonhomogeneous droplets in Figure 2a4).

The oil or organic fraction, $f_{\mathrm{o}}$, as emulsion composition variable, was found to critically affect the stability of the system. Emulsions with varying $f_{\mathrm{o}}$ and 12\% PLA (Table S1) were tested in relation to the emulsion characteristics and ensuing quality of the 3D-printed objects after drying. This PLA concentration (12 wt \%) was found to be the highest possible to allow a fine emulsion gel. More concentrated oil phases were too viscous, making efficient homogenization challenging. As shown in Figure $\mathbf{2} \mathbf{b}_{1}-\mathbf{b}_{3}$, the microstructure of the formed PLA droplets was similar, with stable droplet sizes in the $1-10 \mu \mathrm{m}$ range. The high viscosity of the oil phase at $12 \mathrm{wt} \%$ PLA concentration resulted in size polydispersity. For $f_{0}=0.4$ (Figure $\left.\mathbf{2} \mathbf{b}_{4}\right)$, large droplets $(<40 \boldsymbol{\mu m})$ were observed with a nonhomogeneous distribution. Significant coalescence of PLA droplets occurred at $f_{\mathrm{o}}=0.5$ (Figure $2 \mathbf{b}_{5}$ ). This can be ascribed to the low emulsifying efficiency in this condition. Moreover, an insufficient inventory of surfactant may have prevented the full coverage of newly generated interfaces, easing a more extensive droplet coalescence. It was noted that although droplet coalescence occurred in the emulsion gels, no macroscopic phase separation was apparent after equilibration for $24 \mathrm{~h}$ at room temperature. This is due to the dense internal structure 
originated from strongly gelled CNF that significantly inhibited or delayed the mobility of the oil phase. In summary, the formed emulsion gels showed no oil leakage, even at high internal phase content, which is beneficial for inks to be used for high-fidelity 3D-printing.

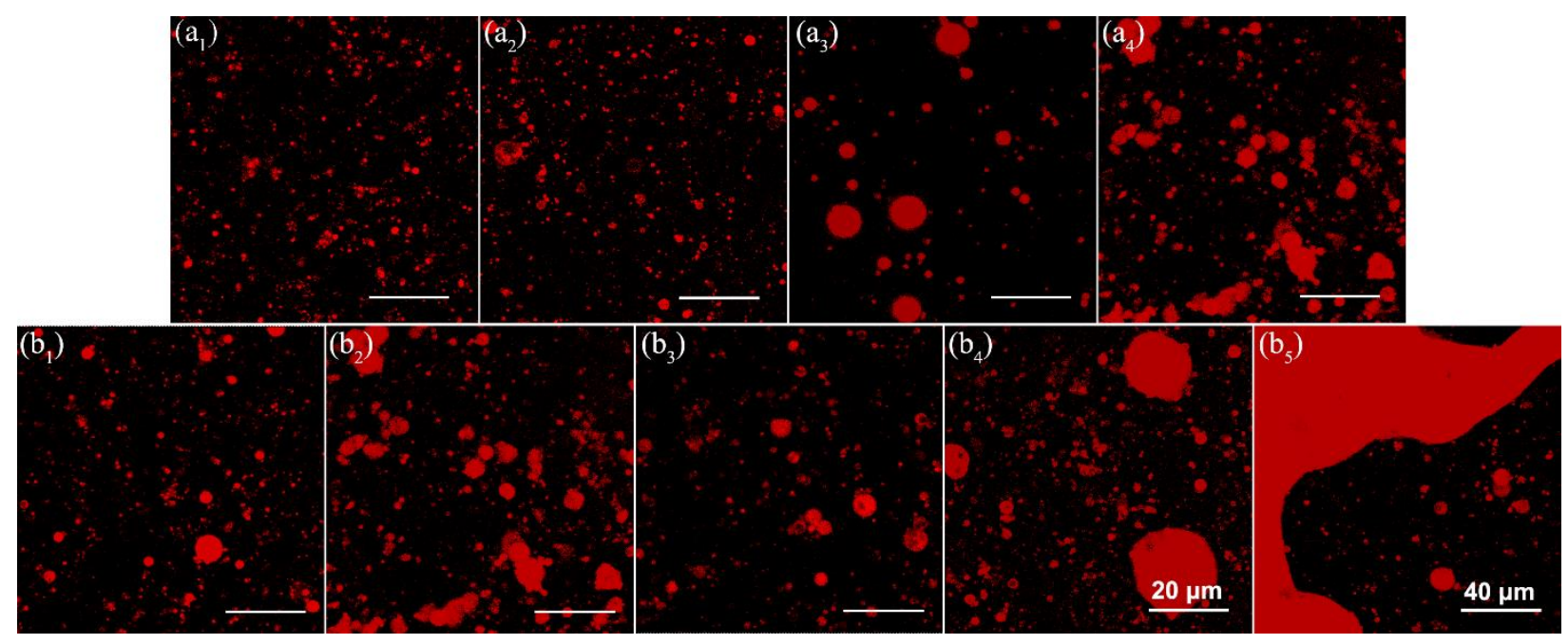

Figure 2. CLSM micrographs of emulsion gels showing the oil phase droplets at $\left(\mathrm{a}_{1}-\mathrm{a}_{4}\right)$ increased concentrations of PLA $\left(0,4,8\right.$, and $12 \mathrm{wt} \%$, respectively) for a $f_{0}=0.2($ WOR $=80: 20)$. Figures $\left(\mathrm{b}_{1}-\mathrm{b}_{5}\right)$ correspond to an increased volume fraction of the internal phase $\left(f_{\mathrm{o}}=0.1,0.2 ., 0.3,0.4\right.$ and 0.5 , respectively). In (a), the $f_{\mathrm{o}}$ for all samples was 0.2 . In (b) the PLA concentration was $12 \mathrm{wt} \%$. The amount of CNF and alginate in the aqueous phase was the same for all the formulations $(1.5 \mathrm{wt} \%$ each, based on the aqueous phase). The oil phase was stained with Nile red. All prepared emulsions were stored at room temperature for $24 \mathrm{~h}$ before imaging. The scale bar corresponds to $20 \mu \mathrm{m}$ in (a) and ( $\left.\mathrm{b}_{1}-\mathrm{b}_{4}\right)$, and the scale bar in $\left(b_{5}\right)$ is $40 \mu \mathrm{m}$.

Rheological properties of the emulsion gels. As shown in Figure 3a and c, all emulsion gels underwent pronounced shear-thinning; the viscosity decreased by several orders of magnitude as the shear rate increased from 0.01 to $100 \mathrm{~s}^{-1}$ (values typically used for DIW). The flow curves of all samples presented similar trends and a similar apparent viscosity was recorded at high shear rates. This result implies that the emulsion viscosity depends mainly on that of the continuous phase. PLA loading in the dispersed phase slightly increased the low-shear viscosity of the emulsion gel but the values at high-shear rates were essentially the same, Figure 3a. This is a very 
useful attribute of the emulsions, e.g., they facilitate transport of high-viscosity dispersed phases while the rheology is mainly dominated by the continuous phase.

Dynamic measurements at low strain (0.5\%) are shown in Figure 3b and d. Within the linear viscoelastic region, and except for emulsions of high internal phase fraction $\left(f_{0}=0.4\right.$ and 0.5$)$, all the emulsion gels presented a $G$ ' plateau value that exceeded $G$ " by about one order of magnitude, indicating a dominant elastic behavior across the entire frequency range. The slight modulus increase at high frequencies for the emulsion systems was a distinctive feature compared to the behavior of other elastic materials. ${ }^{38}$ As shown in Figure 3b, emulsion gels of given PLA concentrations in the organic phase showed similar values of the moduli. The sample containing 4 wt\% PLA presented the largest $G$, which is likely due to the more homogeneous and stable emulsion that was formed. Changes in the internal phase, as measured by the $f_{0}$, induced significant increase in the moduli values, especially $G^{\prime}$, Figure 3d. The $G^{\prime}$ plots displayed two regimes: (1) Emulsion gels of low internal phase fraction $\left(f_{\mathrm{o}}=0.1,0.2\right.$ and 0.3$)$, showing similar frequencydependence with PLA concentration (Figure 3b). In this region $G$ ' presented a maximum value for emulsions with $f_{\mathrm{o}}=0.1$, attributed to the homogeneous dispersion of PLA droplets. (2) Emulsion gels of high dispersed phase fraction $\left(f_{\mathrm{o}}=0.4\right.$ and 0.5$)$, displaying an increased moduli at low frequencies, owing to the partial coalescence of oil droplets that impaired structuring, thereby favoring a more liquid-like behavior. In the sweep cycles, the $G$ " profile displayed a minimum change compared to $G^{\prime}$ (Figure 3d), given that $G^{\prime}$ is more sensitive to oil coalescence at low frequencies. ${ }^{39}$ 

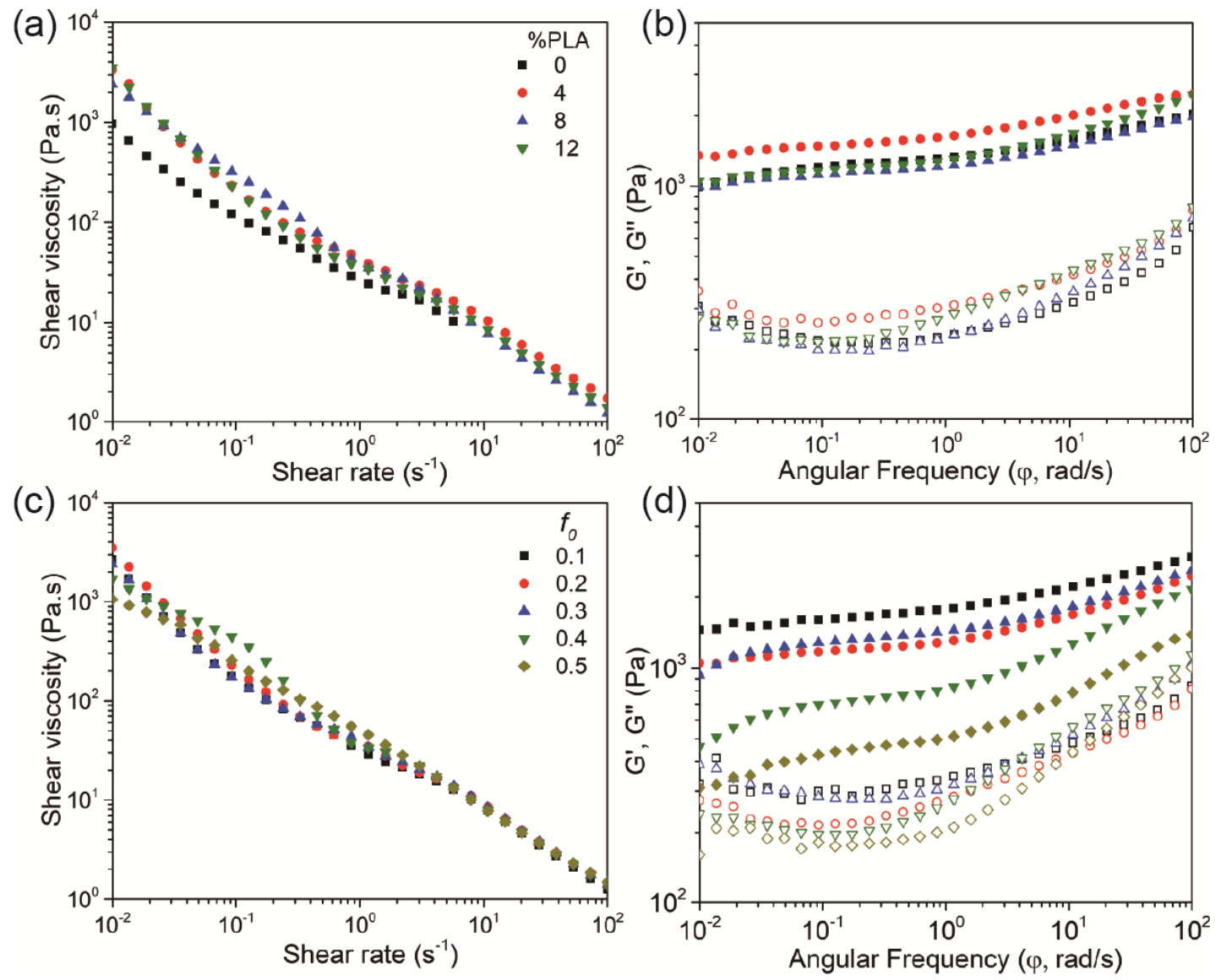

Figure 3. (a) Flow curves with the apparent shear viscosity as a function of shear rate and (b) moduli (storage modulus, $G$ ', and loss modulus, $G$ ") of emulsion gels containing oil droplets with given PLA concentration and $f_{\mathrm{o}}=0.2$. (c) Flow curves and (d) moduli (storage modulus, $G$ ', and loss modulus, $G$ ”) of emulsion gels for different $f_{\mathrm{o}}$ values and PLA concentration of $12 \%$ in the dispersed phase. In (a)-(d) the solid content of CNF and alginate in the aqueous phase was the same (1.5 wt \% each). For (b) and (d), the closed and open symbols correspond to the storage and loss modulus, respectively. All the measurements were performed at constant temperature $\left(25^{\circ} \mathrm{C}\right)$. See Table S1 for nomenclature.

In summary, the emulsion gels developed for direct ink writing exhibited shear-thinning behavior that is expected to enable efficient flow through fine deposition nozzles, yet they should quickly recover as solid-like (sufficiently high storage modulus, $G^{\prime}$ ) after cessation of shear and to maintain its filamentary shape upon printing. ${ }^{40}$ Since alginate shows low zero-shear viscosity, ${ }^{24}$ the presence of CNF in the emulsion gel is beneficial not only as an interfacial stabilizer, but also 
a rheology modifier. The measured rheological properties of the emulsion gels to be used in DIW fit the property space desirable for inks for DIW-based 3D printing ${ }^{41}$ as is discussed next.

DIW and 3D-printed structures. The 3D printing of the emulsion gels is schematically illustrated in Figure 4a and b. Non-crosslinked cubic scaffolds were stable and self-supporting, with no evidence of collapse or surface crumpling after printing (Figure $\mathbf{4} \mathbf{b}_{2}$ ). This confirmed our expectations for DIW requirements based on the rheological behavior of CNF/alginate-based emulsions. The treatment with $\mathrm{Ca}^{2+}$ resulted in the neutralization of the acidic groups and chelation ${ }^{42}$ with alginate and alginate-CNF (Figure $\left.\mathbf{4} \mathbf{b}_{3}\right)$. This allowed a robust internal structure even in the hydrated state (Figure $\mathbf{4 c}$ and Figure $\mathbf{S} \mathbf{3}_{\mathbf{a}}$ ) and improved the integrity of the printed objects (Figure 4e). Furthermore, freeze-drying resulted in objects that retained the shape and size of the original designs, with no collapse, deformation nor shrinkage (Figure $4 \mathbf{b}_{4}$ ) (see also Figure $\mathbf{4 d}$ and Figure $\mathbf{S} 3_{\mathbf{b}}$ ). As shown in Figure $\mathbf{4 f}$, printed layers can be clearly identified from the side view of enlarged letter "A". To further demonstrate the versatility of the emulsion gel ink, a more complex and asymmetrical structure was printed (see Figure S4). The shape and fidelity of the objects were maintained both in the hydrated state and after freeze-drying. In sum, the emulsion gels developed here are efficient 3D printable inks that retain the solid shape and enable precise printing process and structures with good fidelity. 


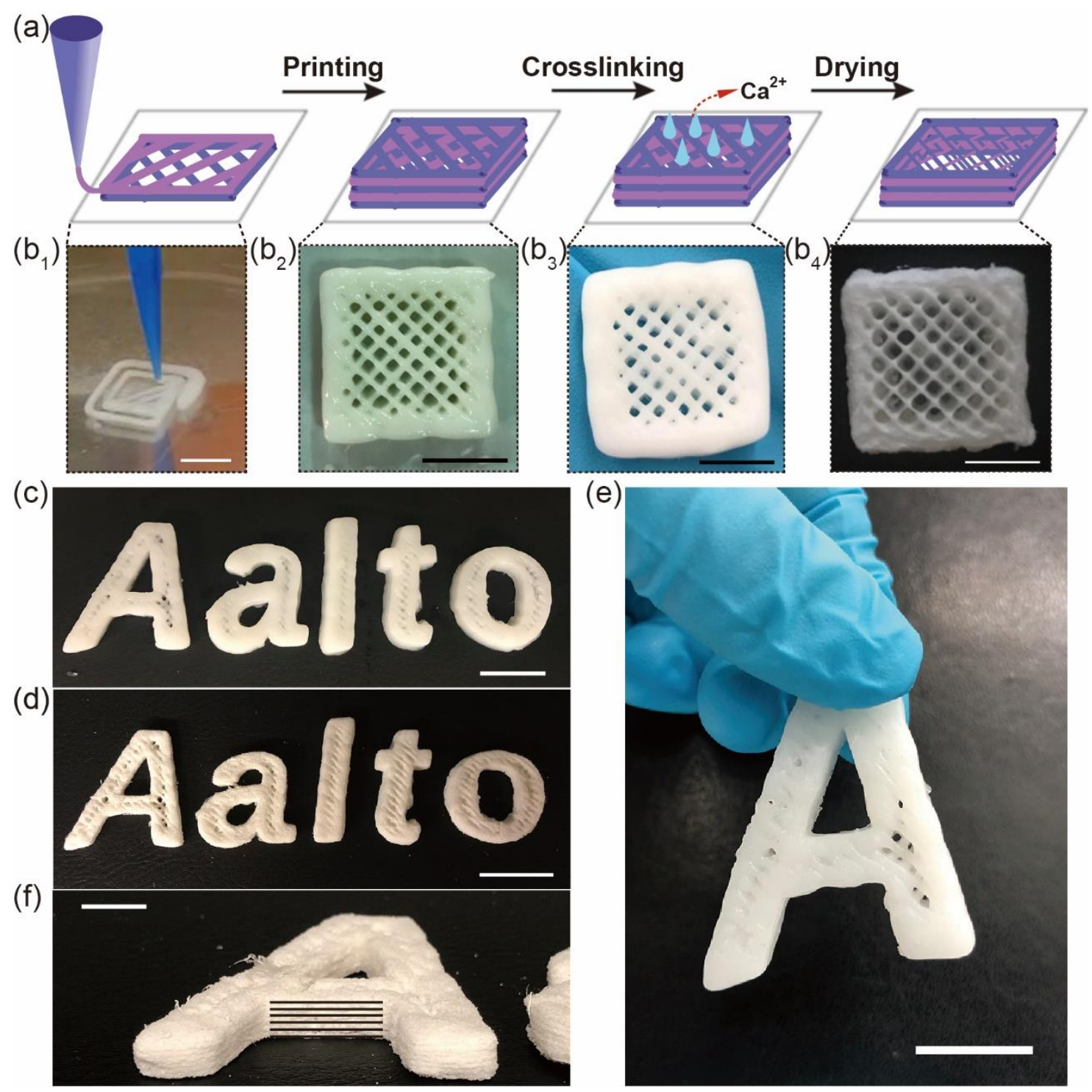

Figure 4. (a) Schematic illustration of the 3D-printing process, crosslinking and drying of CNF-based emulsion gels into cubic scaffolds. $\left(b_{1}\right)$ Emulsion gel is extruded through a nozzle $(0.63 \mathrm{~mm})$ (scale bar is $5 \mathrm{~mm}$ ) onto a PDMF surface to produce a $\left(b_{2}\right)$ cubic scaffold. $\left(b_{3}\right)$ The printed object is crosslinked by immersion in $\mathrm{CaCl}_{2}$ solution $(1.0 \mathrm{wt} \%)$. ( $\left.\mathrm{b}_{4}\right)$ The scaffold is dried to form strong cryogels and xerogels. The scale bar in $\left(b_{2}\right),\left(b_{3}\right)$ and $\left(b_{4}\right)$ is $1 \mathrm{~cm}$. Top view of 3D-printed "letters" after (c) crosslinking and (d) freezedrying. (e) A printed " $A$ " held by hand in the hydrated state soon after crosslinking. The scale bar in (c), (d) and (e) is $2.5 \mathrm{~cm}$. (f) Side view of the printed " $A$ " after freeze-drying. The scale bar is $1 \mathrm{~cm}$. The lines drew in (f) follow five printed layers. The formulation in (b) to (f) was identical: CNF and alginate in equal 
concentrations in the continuous, aqueous phase (1.5 wt $\%$ each). The PLA concentration was $12 \mathrm{wt} \%$ and $f_{\mathrm{o}}=0.3$.

Microstructure of 3D-printed scaffolds. The microstructure of the lines in the printed scaffolds after freeze-drying at various PLA concentrations is displayed in Figure 5a. No clear boundaries between the different layers can be discern in the top view of dried scaffolds (Figure $\mathbf{5}_{\mathbf{a} 1}$ to $\mathbf{a} 4$ ), although the initial layer-by-layer structure was observed visually in the dried objects (see also Figure $\mathbf{4}_{\mathbf{f}}$ and Figure $\mathbf{S 3}$ ). This indicates that the properties of the emulsion ink allowed a good supported structure but did not prevent integration of the layers during printing nor crosslinking. The thickness of the scaffold wall and the surface roughness increased at increased PLA concentration (higher solids) (Figure 5a to $\mathbf{a}_{4}$, inserts). Thus, the incorporation of PLA significantly altered the properties of printed scaffolds and improved their integrity (Figure 5a 1 ). As shown in Figure 5a $\mathbf{a}_{2}$ to $\mathbf{a}_{4}$, layered structures are evident in the inner sections of the dried scaffolds, indicating that the crosslinked shape formed by the emulsion gel was transferred to the cryogel, keeping the pore spaces. Such pores were caused by the initial network that was formed and by the entanglement of $\mathrm{CNF} /$ alginate in the aqueous phase.

Interestingly, small spherical particles were observed on the surface of the walls of the 3Dprinted objects, more evident as PLA loading in the emulsion gels was increased (Figure 5a2 to a4, insets). It can be reasonably proposed that these are PLA particles that formed during freezedrying, given that PLA formed the initial emulsion droplets. Figure S5a 1 to $\mathbf{a}_{4}$ indicates that PLA particles were present in the internal sections of the dried scaffolds, and their corresponding size increased with increasing PLA concentration. It should be noted that most of the PLA particles were trapped or embedded between the lamellar structure of $\mathrm{CNF} /$ alginate. The interactions between PLA and CNF/alginate likely involve structural entanglement. Moreover, the existence 
interfacial adhesion between PLA and CNF cannot be ruled out given the effect of the surfactant, which may act as interfacial compatibilizer, as has been demonstrated for other systems in our previous work involving measurement of the adhesion. ${ }^{43,44}$ The non-adsorbing CNF and alginate originally present in the aqueous phase stabilized the emulsion and formed a porous, layered skeleton upon drying. Moreover, the freely and randomly distributed PLA particles on and inside the $\mathrm{CNF} /$ alginate porous structure acted as support of the internal structure, which resulted in strong scaffolds.

The microstructures of printed scaffolds made from emulsion gels of varying organic phase fraction, $f_{0}$, are shown in Figure 5b. As observed from the top view of the dried scaffolds (Figure $\mathbf{5 b}_{\mathbf{1}}$ to $\left.\mathbf{b}_{5}\right)$, the thickness of the wall increased accordingly. Higher surface roughness and defective structures developed at $f_{\mathrm{o}}=0.4$ and 0.5 The increase in thickness of the scaffold walls is ascribed to the increased loading of PLA, leading to a higher solidity. The change in surface morphology is likely caused by the difference in droplet microstructure in the emulsion gel as a function of the disperse phase fraction $f_{\mathrm{o}}$ (Figure 2b). Smooth solids structures with PLA distributed randomly inside and on the layers were produced from emulsion gels with stable PLA droplets (Figure $\mathbf{2} \mathbf{b}_{1}$ to $\mathbf{b}_{3}$ ). At $f_{0}=0.4$ and 0.5 , the coalescence of PLA droplets in the emulsion gel led to a nonhomogeneous distribution of PLA (Figure $2 \mathbf{b}_{\mathbf{4}}$ and $\mathbf{b}_{5}$ ), a result of unevenly and discontinuously distributed material (surface crumpling and uneven layering upon drying). Furthermore, extensive PLA aggregation was observed in the internal sections of the dried scaffolds (Figure $\mathbf{S 5} \mathbf{b}_{\mathbf{4}}$ and $\mathbf{b}_{\mathbf{5}}$ ), explained by the coalescence of PLA droplets in the emulsion gel as the internal phase concentration increased. From the microstructure of the printed scaffolds (Figure 5), it can be concluded that PLA loading was optimum in emulsion gels prepared with $12 \mathrm{wt} \%$ PLA at $f_{\mathrm{o}}=0.3$ (see also Figure 6). 


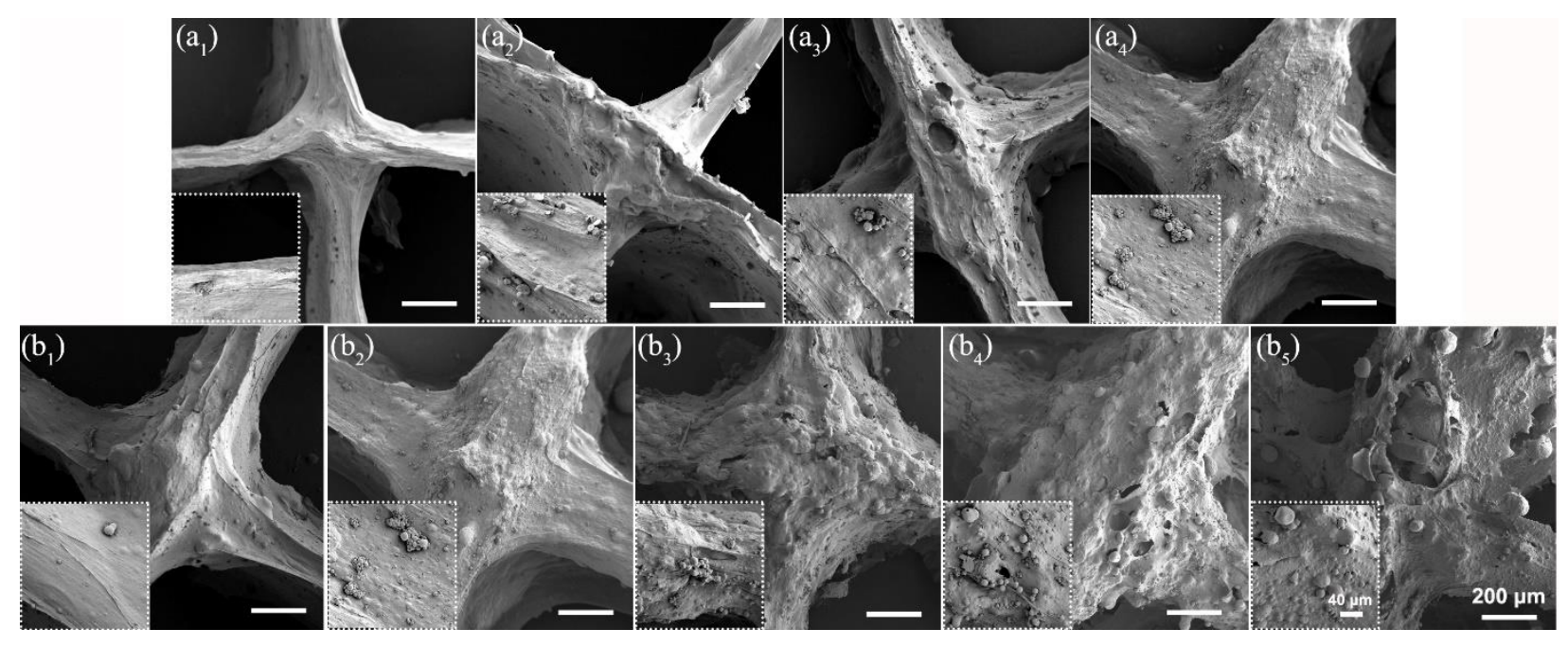

Figure 5. Top view SEM images of 3D-printed orthogonal or crossing lines of freeze-dried scaffolds prepared from emulsion gels of $\left(\mathrm{a}_{1}-\mathrm{a}_{4}\right)$ increased PLA concentrations $(0,4,8$, and $12 \mathrm{wt} \%$, respectively) with $f_{\mathrm{o}}=0.2$ and $\left(\mathrm{b}_{1}-\mathrm{b}_{5}\right)$ increased volume fraction of the internal phase $f_{\mathrm{o}}=0.1,0.2,0.3,0.4,0.5$, respectively with a PLA concentration of $12 \mathrm{wt} \%$. The amount of CNF and alginate in the aqueous phase was the same for all the formulations ( $1.5 \mathrm{wt} \%$ each, based on the aqueous phase). The insets in (a) and (b) correspond to enlarged surface microstructures. The scale bar in (a) and (b) is $200 \mu \mathrm{m}$, and that in the insets is $40 \boldsymbol{\mu m}$. The internal sections of the dried scaffolds can be seen in the supporting information, Figure S5.

Shrinking. All emulsion gel formulations were printed and crosslinked by $\mathrm{Ca}^{2+}$ to form cubic scaffolds $(2 \times 2 \times 0.5 \mathrm{~cm})$. The appearance and shrinkage of the cubic scaffolds in the plane and out-of-plane directions after drying at room temperature (RT) and freeze-drying are shown in Figure 6 and the shrinkage $\%$ is presented in Table S2. Except for the systems with $f_{\mathrm{o}}=0.4$ and 0.5 , the emulsion gels were easily printed at high fidelity into hydrated scaffolds. In the case of high internal phase systems, the non-homogeneous and coalesced emulsion droplets produced transient, discontinuous extrusion. Significant shrinkage, both from surface and height directions occurred upon room temperature (RT) drying. In contrast, freeze-drying was effective to retain the shape of the printing objects, with negligible shrinkage. The extent of shape retention of all dried scaffolds obtained from freeze-drying is explained by the tightly locked internal structure induced by ice crystal formation upon freezing, which slows down mass transport during water removal. 
For RT-drying, at increased PLA concentration (from 0 to $12 \mathrm{wt} \%$ ), objects were obtained with a dry mass ratio of PLA in the emulsion gel from 0 to $50 \%$ (Table S1). In these systems, the shrinkage $\%$ gradually decreased from 57.5 to $52.5 \%$, reaching a minimum value at 8 wt $\%$ PLA (Table S2). The shrinkage rate decreased to $45 \%$ by increasing PLA loading at $f_{\mathrm{o}}=0.4$ and 0.5 . Since PLA is randomly embedded in the entire scaffold from the respective emulsion gel, it provides a strong resistance to shrinkage during the slow water evaporation at ambient temperature. At lower internal phase concentration $\left(f_{\mathrm{o}}=0.3\right)$, similar shrinkage was observed in the plane and out-of-plane directions (Table S2). Better shape fidelity and microstructure was observed (Figure $5 \mathbf{b 3}$ and 6) in objects produced from the emulsion gels with $12 \mathrm{wt} \%$ PLA and $f_{\mathrm{o}}=0.3$. Compared with other works, ${ }^{20}$ no surface collapse or lateral deformation of the dried scaffolds occurred, indicating that the incorporation of PLA in the emulsion gel limited dryinginduced shape failure. Thus, irregular and uncontrolled destruction of 3D structured objects produced at low CNF solids were prevented. Importantly, incorporation of PLA in the emulsion gels restricted the fusion of printed layers (Figure 5f, $\mathbf{6}$ and $\mathbf{S 3} \mathbf{3}_{\mathbf{b}}$ ), which otherwise occurs in systems formulated with neat CNF. To our knowledge, this level of shape-retention, e.g., prevention of surface collapse or shape deformation, after RT-drying of printed CNF-based objects, has not been achieved to this extent. The results in this study open an opportunity for dryable CNFbased 3D structured materials. 

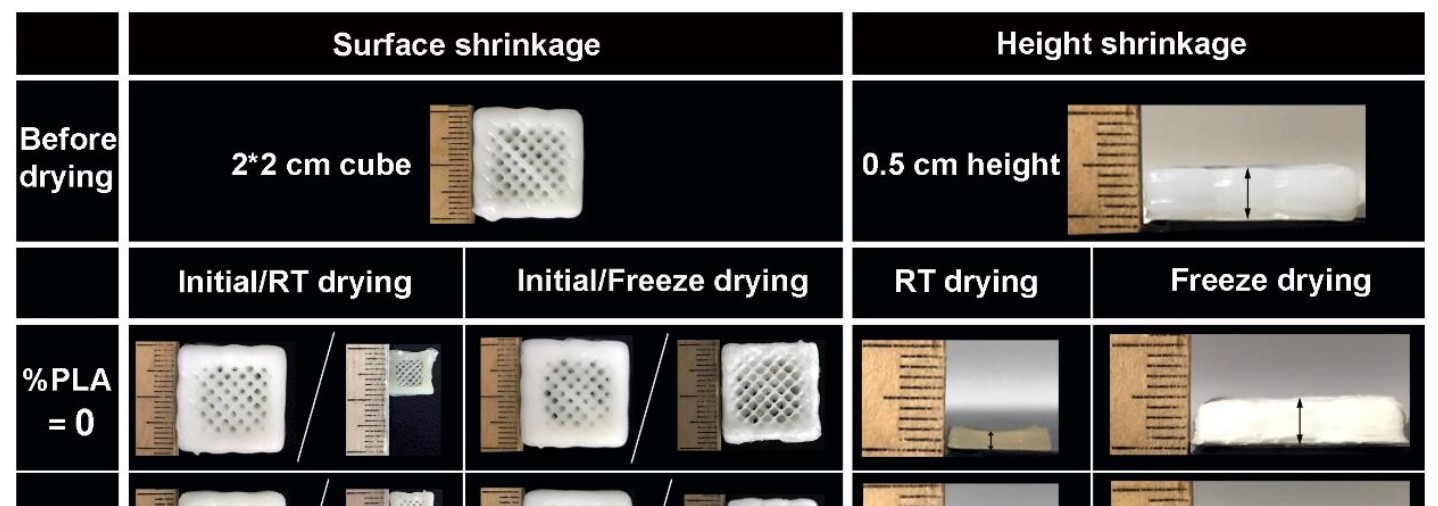

4
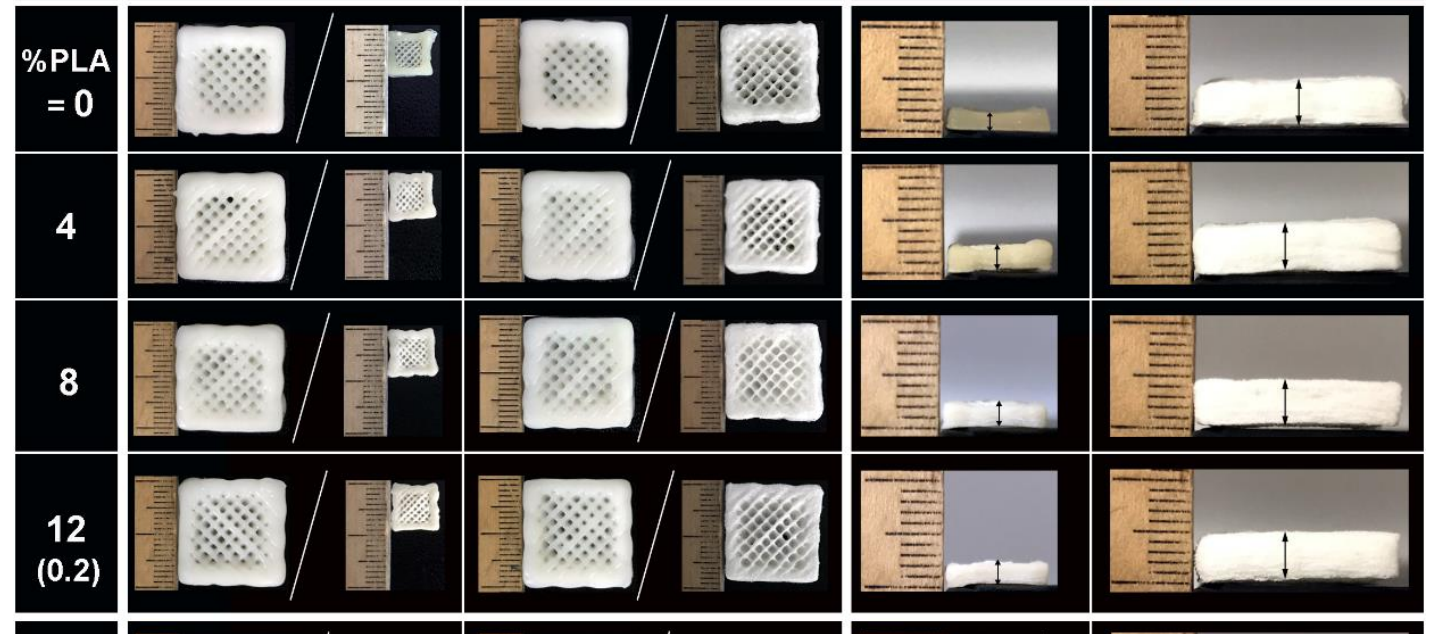

12
$(0.2)$
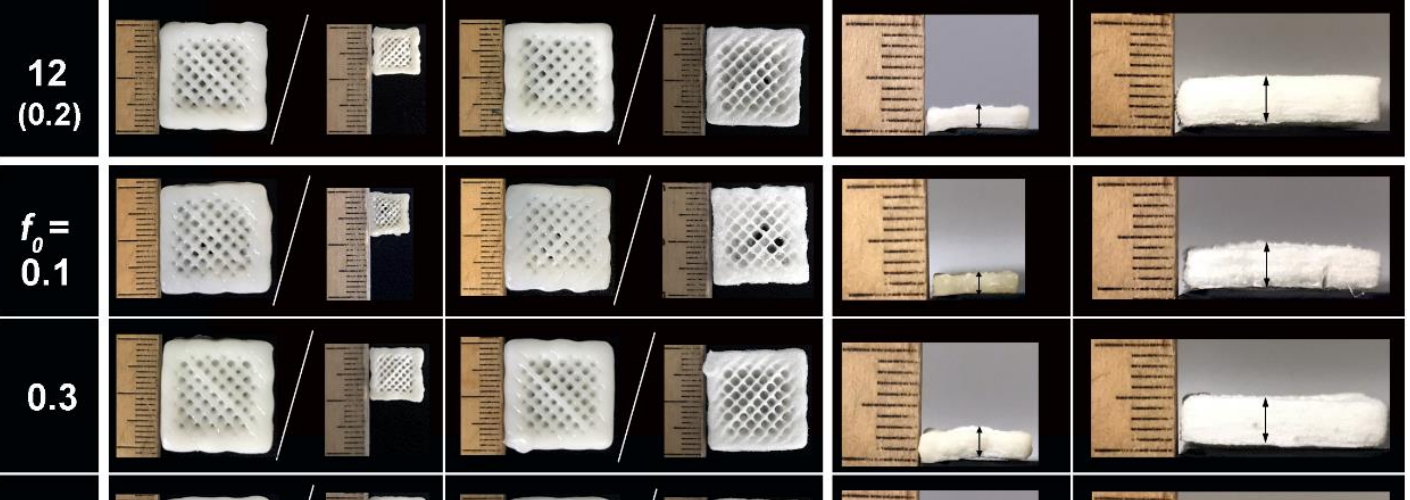

0.4
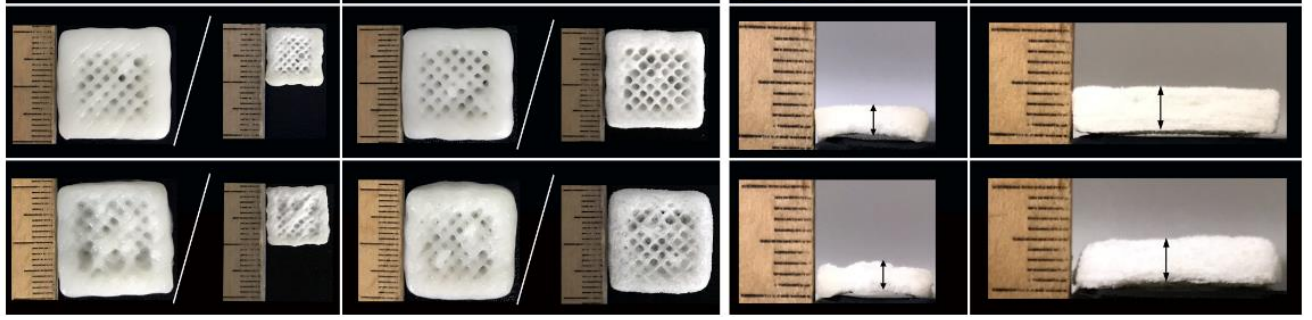

Figure 6. Dried cubic scaffolds 3D-printed from emulsion gels at given PLA concentrations. The shrinkage was calculated both in-plane and out-of-plane (Table S2). References comprising cross-linked hydrated scaffolds were added for every sample. For out-of-plane shrinkage, a single reference was used for all the samples. The hydrated scaffolds were dried at room temperature (RT) and freeze-drying, respectively. Note that an organic phase fraction, $f_{\mathrm{o}}=0.2$ was used for samples shown at varying PLA concentrations while $12 \%$ PLA was used for samples produced at varying $f_{\mathrm{o}}$, as indicated.

Mechanical compression. Many reports have shown that the mechanical integrity of CNFbased hydrogels can be severely compromised upon exposure to humid or aqueous environments, 
due to the disruption of hydrogen bonding. ${ }^{45}$ The incorporation of PLA as a supporting material in a CNF matrix, may provide a simple way to overcome such an issue. As shown in Figure S6a and c, the compression profiles of crosslinked scaffolds show a similar trend for all emulsion gel formulations, with all hydrated scaffolds reaching a flattened structure at high compression $(\sim 80 \%$ compressive strain and $30 \mathrm{KPa}$ stress), confirming that the mechanical strength of the hydrated scaffolds was sufficient for handling (Figure 4e). The freeze-dried scaffolds were significantly affected by PLA loading (Figure S6b and d): the increased PLA dry mass ratio in the emulsion gel resulted in objects with less compression strain (stiffer scaffolds). Since PLA is randomly distributed in the scaffold, increasing PLA provides better CNF skeletal support, improving the mechanical strength. Owing to the constant loading level for all samples (maximum at $18 \mathrm{~N}$ ), the compression stress of the dried scaffolds was similar to that measured for the hydrated ones. Interestingly, a recent study on the properties of CNF-based aerogels, reported a similar compression strain, but at much lower compression stress (maximum $\sim 11 \mathrm{KPa}$ ) ${ }^{46}$ indicating that the incorporation of PLA into CNF/alginate matrix has a positive effect in improving the mechanical performance of the dried scaffolds. Overall, the compression tests indicate that freezedried scaffolds were sufficiently robust to maintain the initial shape.

Swelling behavior. The hydrophilicity of CNF promotes water adsorption by the dried materials, which is a critical consideration for any use. Therefore, the performance of printed objects after contact with aqueous or humid environments (re-hydration) was studied. As shown in Figure 7a,b, the water absorption rate for all scaffolds reached a maximum value in less than 2 h. The swelling of the scaffolds correlated inversely with the PLA content in the emulsion gel, given its lower surface energy (Table S1). A lower saturation swelling at higher PLA loading levels was observed, reaching a minimum absorption at around $400 \%$ at $f_{\mathrm{o}}=0.4$. PLA limited the 
transport and sorption of water even in the presence of CNF and alginate, both of which are quite hydrophilic. ${ }^{47}$ Interestingly, a recent study revealed a swelling rate of $380 \%$ for CNF-stabilized, chemically-crosslinked porous foam made via oven-drying, ${ }^{48}$ which is close to the saturation value of the system at high PLA loading. Accordingly, it can be inferred that the highly porous, inner structure of freeze-dried 3D materials and the internal micro-channels, contribute to high water absorption via capillary and other phenomena. Furthermore, the non-chemically crosslinking feature of 3D printed scaffolds ensure a higher water uptake ability. Remarkably, after immersion in water, no shape changes occurred compared to the initial 3D-printed shape (Figure S7). Thus, although water sorption is relatively extensive, the drying and re-hydrating ability of the printed emulsion gels is an asset, depending on their intended use. For example, they include structural materials that absorb water while being able to support given loads (high elastic modulus) and maintain the shape upon hydration. This is relevant to absorbents that can be functionalized by taking advantage of the convenient modification of the cellulose fraction.
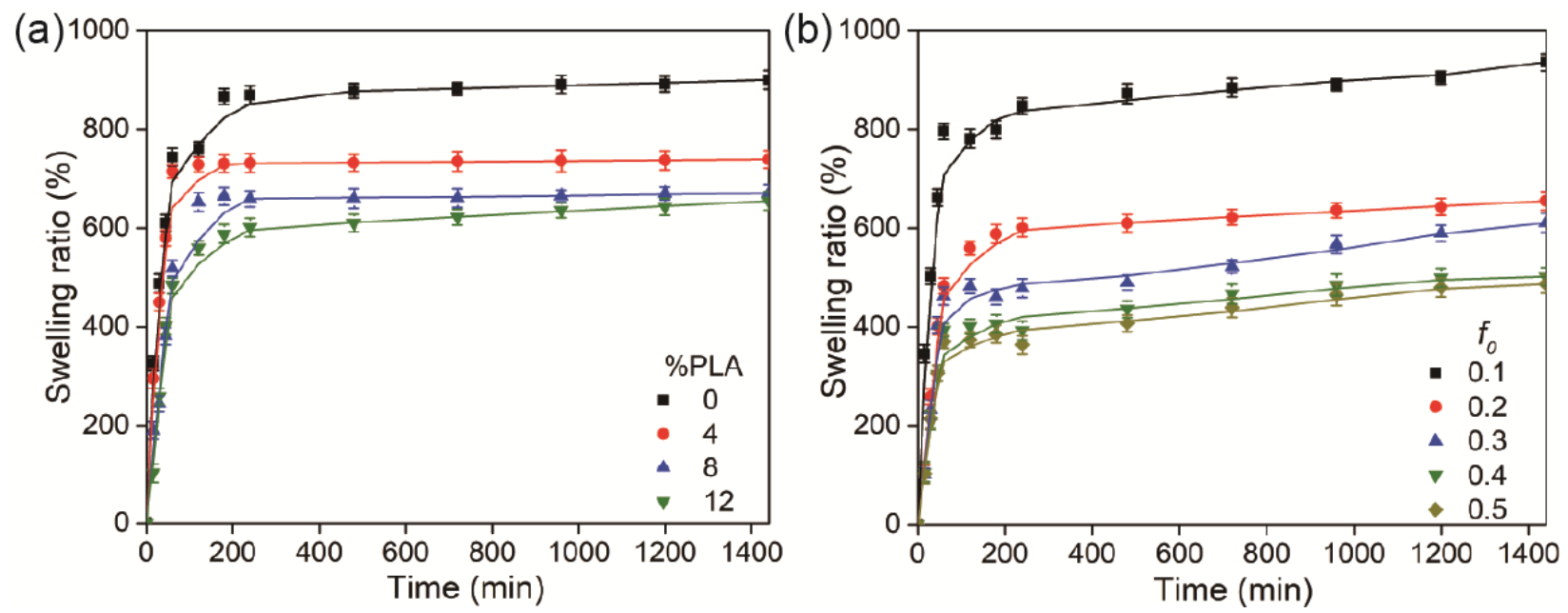

Figure 7. Water swelling profiles of dried cubic scaffolds produced from emulsion gels of given (a) PLA concentrations and (b) organic phase fraction, $f_{0}$. All the measurements were conducted at ambient temperature. 
To finalize, we summarize the contribution of each of the components throughout the 3D printing. Although the viscosity of alginate is considerably high, ${ }^{49}$ it is not sufficient for achieving shape fidelity given its low zero-shear viscosity. ${ }^{24}$ Thus, it is used here mainly for crosslinking. In turn, the nanocellulose is a critical component to ensure the printability of the ink and the corresponding structural fidelity. ${ }^{26}$ PLA improves the printability of the ink at low solid content; ${ }^{50}$ supports static load in the hydrated state; enhances the performance of the printed objects and regulates the interaction with water of the dry objects. These components can only be incorporated in an emulsion ink that allows control on the viscosity, mainly affected by the continuous phase and drop size.

\section{CONCLUSIONS}

We demonstrate a simple and efficient method based on emulsion gels to synthesize low-solid 3D scaffolds containing CNF and alginate via direct ink writing. PLA added to the dispersed phase was used to tailor the properties of the solid materials. Simple cubic scaffolds and complex geometric structures were built with high shape fidelity. The microstructure of the printed scaffolds were finely tuned by addition of PLA through randomly embedding particles in the CNF/alginate system. PLA also acted as internal enhancer to mechanically support the inner structure of the printed layers. The shrinking of the scaffolds was limited, showing no collapse nor deformation relative to the initial shape. The swelling behavior of dried scaffolds demonstrate their robustness as a promising candidate for application in the presence of water. Our findings are relevant to the application of biobased materials in 3D-printable inks. The proposed method presents a new way to construct low-solids, dryable, and swellable CNF-based 3D structured objects, which can be readily transferred to applications that require biocompatibility, including biomedical devices. 


\section{ASSOCIATED CONTENT}

Supporting Information. Additional materials are provided including confocal images of emulsion gels with various CNF-to-alginate ratios; visual appearance of emulsion gels cast in round plates after crosslinking; side view of printed objects; top view of a printed "Ear"; SEM images of enlarged internal microstructure of printed objects; mechanical performance of printed cubic scaffolds; visual appearance of scaffold after swelling test; a table for dry mass ratio of CNF/alginate to PLA; and a table for detailed shrinkage rate of printed scaffolds.

\section{AUTHOR INFORMATION}

\section{Corresponding Authors}

* E-mail: orlando.rojas@aalto.fi (O.J.R.), Tel: +358-(0)50 512 4227;

*E-mail: long.bai@aalto.fi (L.B.), Tel: +358-(0)50 3394495

\section{Author Contributions}

The manuscript was written through contributions of all authors. All authors have given approval to the final version of the manuscript.

\section{ACKNOWLEDEGMENTS}

We acknowledge funding support by the Academy of Finland's Biofuture 2025 program under project 2228357-4, 3D-Manufacturing of Novel Biomaterials. The authors are also grateful for the support by the European Research Commission (O.J.R.'s ERC Advanced Grant) and the Academy of Finland through Centers of Excellence Program (2014-2019) under Project 264677 "Molecular Engineering of Biosynthetic Hybrid Materials Research” (HYBER). 


\section{REFERENCES}

(1) Gladman, A. S.; Matsumoto, E. A.; Nuzzo, R. G.; Mahadevan, L.; Lewis, J. A. Biomimetic 4D printing. Nat. Mater. 2016, 15, 413.

(2) Murphy, S. V.; Atala, A. 3D bioprinting of tissues and organs. Nat. Biotechnol. 2014, 32, 773.

(3) Wang, B.; Benitez, A. J.; Lossada, F.; Merindol, R.; Walther, A. Bioinspired mechanical gradients in cellulose nanofibril/polymer nanopapers. Angew. Chem. Int. Ed. 2016, 128, 60706074.

(4) Hong, S.; Sycks, D.; Chan, H. F.; Lin, S.; Lopez, G. P.; Guilak, F.; Leong, K. W.; Zhao, X. 3D printing of highly stretchable and tough hydrogels into complex, cellularized structures. Adv. Mater. 2015, 27, 4035-4040.

(5) Ngo, T. D.; Kashani, A.; Imbalzano, G.; Nguyen, K. T.; Hui, D. Additive manufacturing (3D printing): A review of materials, methods, applications and challenges. Composites, Part B 2018, $143,172-196$.

(6) Odent, J.; Wallin, T. J.; Pan, W.; Kruemplestaedter, K.; Shepherd, R. F.; Giannelis, E. P. Highly Elastic, Transparent, and Conductive 3D-Printed Ionic Composite Hydrogels. Adv. Funct. Mater. 2017, 27.

(7) Appel, E. A.; Tibbitt, M. W.; Webber, M. J.; Mattix, B. A.; Veiseh, O.; Langer, R. Selfassembled hydrogels utilizing polymer-nanoparticle interactions. Nat. Commun. 2015, 6, 6295.

(8) Lewis, J. A. Direct ink writing of 3D functional materials. Adv. Funct. Mater. 2006, 16, 2193 2204.

(9) Rauzan, B. M.; Nelson, A. Z.; Lehman, S. E.; Ewoldt, R. H.; Nuzzo, R. G. Particle-Free Emulsions for 3D Printing Elastomers. Adv. Funct. Mater. 2018, DOI: 10.1002/adfm.201707032.

(10) Thakkar, H.; Eastman, S.; Al-Naddaf, Q.; Rownaghi, A. A.; Rezaei, F. 3D-Printed MetalOrganic Framework Monoliths for Gas Adsorption Processes. ACS Appl. Mater. Interfaces 2017, 9, 35908-35916.

(11) Gratson, G. M.; Xu, M.; Lewis, J. A. Microperiodic structures: direct writing of threedimensional webs. Nature 2004, 428, 386. 
(12) Siqueira, G.; Kokkinis, D.; Libanori, R.; Hausmann, M. K.; Gladman, A. S.; Neels, A.; Tingaut, P.; Zimmermann, T.; Lewis, J. A.; Studart, A. R. Cellulose nanocrystal inks for 3D printing of textured cellular architectures. Adv. Funct. Mater. 2017, 27, 1604619.

(13) Minas, C.; Carnelli, D.; Tervoort, E.; Studart, A. R. 3D printing of emulsions and foams into hierarchical porous ceramics. Adv. Mater. 2016, 28, 9993-9999.

(14) Sommer, M. R.; Alison, L.; Minas, C.; Tervoort, E.; Rühs, P. A.; Studart, A. R. 3D printing of concentrated emulsions into multiphase biocompatible soft materials. Soft Matter 2017, 13, 1794-1803.

(15) Zhou, N.; Liu, C.; Lewis, J. A.; Ham, D. Gigahertz Electromagnetic Structures via Direct Ink Writing for Radio - Frequency Oscillator and Transmitter Applications. Adv. Mater. 2017, 29.

(16) Xu, W.; Wang, X.; Sandler, N.; Willfor, S.; Xu, C. Three-dimensional printing of woodderived biopolymers: A review focused on biomedical applications. ACS Sustainable Chem. Eng. 2018, 6, 5663-5680.

(17) Xu, X.; Wang, H.; Jiang, L.; Wang, X.; Payne, S. A.; Zhu, J.; Li, R. Comparison between cellulose nanocrystal and cellulose nanofibril reinforced poly (ethylene oxide) nanofibers and their novel shish-kebab-like crystalline structures. Macromolecules 2014, 47, 3409-3416.

(18) Xu, X.; Liu, F.; Jiang, L.; Zhu, J.; Haagenson, D.; Wiesenborn, D. P. Cellulose nanocrystals vs. cellulose nanofibrils: a comparative study on their microstructures and effects as polymer reinforcing agents. ACS Appl. Mater. Interfaces 2013, 5, 2999-3009.

(19) Ávila, H. M.; Schwarz, S.; Rotter, N.; Gatenholm, P. 3D bioprinting of human chondrocyteladen nanocellulose hydrogels for patient-specific auricular cartilage regeneration. Bioprinting 2016, $1,22-35$.

(20) Håkansson, K. M.; Henriksson, I. C.; de la Peña Vázquez, C.; Kuzmenko, V.; Markstedt, K.; Enoksson, P.; Gatenholm, P. Solidification of 3D printed nanofibril hydrogels into functional 3D cellulose structures. Adv. Mater. Technol. 2016, 1.

(21) Li, V. C.; Mulyadi, A.; Dunn, C. K.; Deng, Y.; Qi, H. J. Direct Ink Write 3D Printed Cellulose Nanofiber Aerogel Structures with Highly Deformable, Shape Recoverable, and 
Functionalizable Properties. ACS Sustainable Chem. Eng. 2018, 6, 2011-2022.

(22) Syverud, K.; Pettersen, S. R.; Draget, K.; Chinga-Carrasco, G. Controlling the elastic modulus of cellulose nanofibril hydrogels-scaffolds with potential in tissue engineering. Cellulose 2015, 22, 473-481.

(23) Chinga-Carrasco, G. Potential and Limitations of Nanocelluloses as Components in Biocomposite Inks for Three-Dimensional Bioprinting and for Biomedical Devices. Biomacromolecules 2018, 19, 701-711.

(24) Markstedt, K.; Mantas, A.; Tournier, I.; Martínez Ávila, H.; Hägg, D.; Gatenholm, P. 3D bioprinting human chondrocytes with nanocellulose-alginate bioink for cartilage tissue engineering applications. Biomacromolecules 2015, 16, 1489-1496.

(25) Nguyen, D.; Hägg, D. A.; Forsman, A.; Ekholm, J.; Nimkingratana, P.; Brantsing, C.; Kalogeropoulos, T.; Zaunz, S.; Concaro, S.; Brittberg, M. Cartilage tissue engineering by the 3D bioprinting of iPS cells in a nanocellulose/alginate bioink. Sci. Rep. 2017, 7, 658.

(26) Leppiniemi, J.; Lahtinen, P.; Paajanen, A.; Mahlberg, R.; Metsä-Kortelainen, S.; Pinomaa, T.; Pajari, H.; Vikholm-Lundin, I.; Pursula, P.; Hytönen, V. P. 3D-Printable Bioactivated Nanocellulose-Alginate Hydrogels. ACS Appl. Mater. Interfaces 2017, 9, 21959-21970.

(27) Håkansson, K. M.; Fall, A. B.; Lundell, F.; Yu, S.; Krywka, C.; Roth, S. V.; Santoro, G.; Kvick, M.; Wittberg, L. P.; Wågberg, L. Hydrodynamic alignment and assembly of nanofibrils resulting in strong cellulose filaments. Nat. Commun. 2014, 5, 4018.

(28) Shin, S.; Park, S.; Park, M.; Jeong, E.; Na, K.; Youn, H. J.; Hyun, J. Cellulose Nanofibers for the Enhancement of Printability of Low Viscosity Gelatin Derivatives. BioResources 2017, 12, 2941-2954.

(29) Huan, S.; Liu, G.; Cheng, W.; Han, G.; Bai, L. Electrospun Poly (lactic acid)-Based Fibrous Nanocomposite Reinforced by Cellulose Nanocrystals: Impact of Fiber Uniaxial Alignment on Microstructure and Mechanical Properties. Biomacromolecules 2018, 19, 1037-1046.

(30) Bai, L.; Greca, L. G.; Xiang, W.; Lehtonen, J.; Huan, S.; Nugroho, R. W. N.; Tardy, B. L.; Rojas, O. J. Adsorption and assembly of cellulosic and lignin colloids at oil/water interfaces. Langmuir 2018, DOI:10.1021/acs.langmuir.8b01288. 
(31) Gestranius, M.; Stenius, P.; Kontturi, E.; Sjöblom, J.; Tammelin, T. Phase behaviour and droplet size of oil-in-water Pickering emulsions stabilised with plant-derived nanocellulosic materials. Colloids Surf., A 2017, 519, 60-70.

(32) Huan, S.; Yokota, S.; Bai, L.; Ago, M.; Borghei, M.; Kondo, T.; Rojas, O. J. Formulation and Composition Effects in Phase Transitions of Emulsions Costabilized by Cellulose Nanofibrils and an Ionic Surfactant. Biomacromolecules 2017, 18, 4393-4404.

(33) Zhang, Y.; Wu, J.; Wang, B.; Sui, X.; Zhong, Y.; Zhang, L.; Mao, Z.; Xu, H. Cellulose nanofibril-reinforced biodegradable polymer composites obtained via a Pickering emulsion approach. Cellulose 2017, 24, 3313-3322.

(34) Bai, L.; Huan, S.; Xiang, W.; Rojas, O. J. Pickering emulsions by combining cellulose nanofibrils and nanocrystals: Phase behavior and depletion stabilization. Green Chem. 2018, 20, 1571-1582.

(35) Guo, J.; Filpponen, I.; Su, P.; Laine, J.; Rojas, O. J. Attachment of gold nanoparticles on cellulose nanofibrils via click reactions and electrostatic interactions. Cellulose 2016, 23, 3065 3075 .

(36) Wang, S.; Kempen, D. H.; Simha, N. K.; Lewis, J. L.; Windebank, A. J.; Yaszemski, M. J.; Lu, L. Photo-cross-linked hybrid polymer networks consisting of poly (propylene fumarate) and poly (caprolactone fumarate): controlled physical properties and regulated bone and nerve cell responses. Biomacromolecules 2008, 9, 1229-1241.

(37) Bai, L.; Liu, F.; Xu, X.; Huan, S.; Gu, J.; McClements, D. J. Impact of polysaccharide molecular characteristics on viscosity enhancement and depletion flocculation. J. Food Eng. 2017, 207, 35-45.

(38) Brummer, R. Rheology essentials of cosmetic and food emulsions, Springer Science \& Business Media: 2006.

(39) Huang, S.; Liu, Z.; Yin, C.; Wang, Y.; Gao, Y.; Chen, C.; Yang, M. Enhancement effect of filler network on isotactic polypropylene/carbon black composite melts. Colloid. Polym. Sci. 2011, 289, 1673-1681.

(40) Compton, B. G.; Lewis, J. A. 3D-printing of lightweight cellular composites. Adv. Mater. 2014, 26, 5930-5935. 
(41) Li, Y.; Gao, T.; Yang, Z.; Chen, C.; Luo, W.; Song, J.; Hitz, E.; Jia, C.; Zhou, Y.; Liu, B. 3DPrinted, All-in-One Evaporator for High-Efficiency Solar Steam Generation under 1 Sun Illumination. Adv. Mater. 2017, 29.

(42) Russo, R.; Malinconico, M.; Santagata, G. Effect of cross-linking with calcium ions on the physical properties of alginate films. Biomacromolecules 2007, 8, 3193-3197.

(43) Khakalo, A.; Filpponen, I.; Rojas, O. J. Protein-mediated interfacial adhesion in composites of cellulose nanofibrils and polylactide: Enhanced toughness towards material development. Compos. Sci. Technol. 2018, 160, 145-151.

(44) Khakalo, A.; Filpponen, I.; Rojas, O. J. Protein adsorption tailors the surface energies and compatibility between polylactide and cellulose nanofibrils. Biomacromolecules 2017, 18, 14261433.

(45) Hossen, M. R.; Dadoo, N.; Holomakoff, D. G.; Co, A.; Gramlich, W. M.; Mason, M. D. Wet stable and mechanically robust cellulose nanofibrils (CNF) based hydrogel. Polymer 2018, 151, 231-241.

(46) Guo, L.; Chen, Z.; Lyu, S.; Fu, F.; Wang, S. Highly flexible cross-linked cellulose nanofibril sponge-like aerogels with improved mechanical property and enhanced flame retardancy.

Carbohydr. Polym. 2018, 179, 333-340.

(47) Zimmermann, M. V.; Borsoi, C.; Lavoratti, A.; Zanini, M.; Zattera, A. J.; Santana, R. M. Drying techniques applied to cellulose nanofibers. J. Reinf. Plast. Compos. 2016, 35, 628-643.

(48) Cervin, N. T.; Johansson, E.; Larsson, P. A.; Wågberg, L. Strong, water-durable, and wetresilient cellulose nanofibril-stabilized foams from oven drying. ACS Appl. Mater. Interfaces 2016, 8, 11682-11689.

(49) Kong, H.-J.; Lee, K. Y.; Mooney, D. J. Decoupling the dependence of rheological/mechanical properties of hydrogels from solids concentration. Polymer 2002, 43, 6239-6246.

(50) Dong, J.; Li, M.; Zhou, L.; Lee, S.; Mei, C.; Xu, X.; Wu, Q. The influence of grafted cellulose nanofibers and postextrusion annealing treatment on selected properties of poly (lactic acid) filaments for 3D printing. J. Polym. Sci., Part B: Polym. Phys. 2017, 55, 847-855. 
For Table of Contents Use Only

Low solids emulsion gels based on nanocellulose for 3D-printing Siqi Huan ${ }^{1}$, Rubina Ajdary ${ }^{1}$, Long Bai ${ }^{1, *}$, Ville Klar ${ }^{2}$, Orlando J. Rojas ${ }^{1,{ }^{*}}$
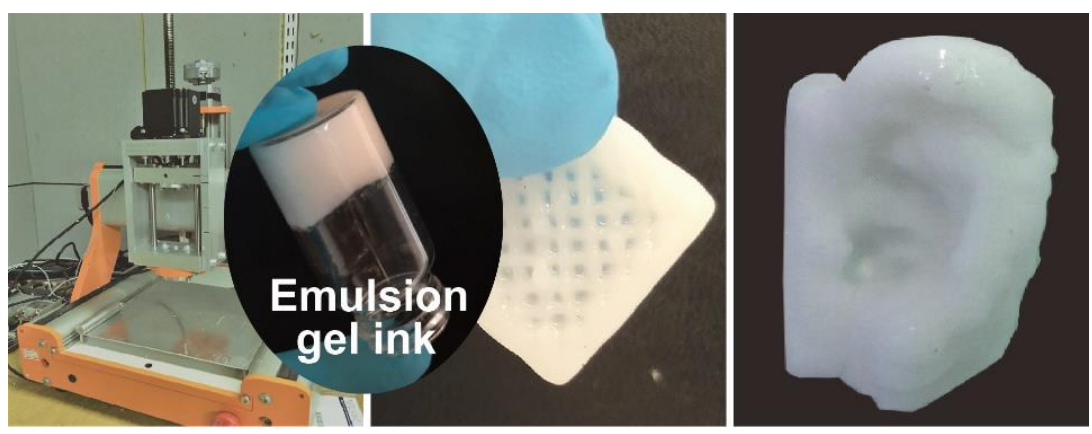